\title{
BMJ Open Psychiatric hospital reform in low- income and middle-income countries Structured Individualised inTervention And Recovery (SITAR): a two-arm pragmatic randomised controlled trial study protocol
}

\author{
Tasneem Raja (D) , ${ }^{1,2}$ Helena Tuomainen (D) , ${ }^{2}$ Jason Madan, ${ }^{3}$ Dipesh Mistry, ${ }^{4}$ \\ Sanjeev Jain, ${ }^{5}$ Swaran Singh (i) ${ }^{6}$
}

To cite: Raja T, Tuomainen $\mathrm{H}$, Madan J, et al. Psychiatric hospital reform in low-income and middle-income countries Structured Individualised inTervention And Recovery (SITAR): a two-arm pragmatic randomised controlled trial study protocol. BMJ Open 2020;10:e035753. doi:10.1136/ bmjopen-2019-035753

- Prepublication history for this paper is available online. To view these files, please visit the journal online (http://dx.doi org/10.1136/bmjopen-2019035753).

Received 18 November 2019 Revised 14 March 2020 Accepted 19 March 2020
Check for updates

(C) Author(s) (or their employer(s)) 2020. Re-use permitted under CC BY-NC. No commercial re-use. See rights and permissions. Published by BMJ.

For numbered affiliations see end of article.

Correspondence to

Ms Tasneem Raja;

T.Raja@warwick.ac.uk

\section{ABSTRACT}

Introduction Low-income and middle-income settings like India have large treatment gaps in mental healthcare. People with severe mental disorders face impediments to their clinical and functional recovery, and have large unmet needs. The infrastructure and standards of care are poor in colonial period psychiatric hospitals, with no clear pathways to discharge and successfully integrate recovered individuals into the community. Our aim is to study the impact of psychiatric hospital reform on individual patient outcomes in a psychiatric hospital in India.

Methods and analysis Structured Individualised inTervention And Recovery (SITAR) is a two-arm pragmatic randomised controlled trial, focusing on patients aged 18-60 years with a hospital stay of 12-120 months and a primary diagnosis of psychosis. It tests the effectiveness of structural and process reform with and without an individually tailored recovery plan on patient outcomes of disability (primary outcome WHO Disability Assessment Scale), symptom severity, social and occupational functioning and quality of life. A computer-generated permuted block randomisation schedule will allocate recruited subjects to the two study arms. We aim to recruit 100 people into each trial arm. Baseline and outcome measures will be undertaken by trained researchers independent to the case managers providing the individual intervention. A health economic analysis will determine the costing of implementing the individually tailored recovery plan.

Ethics and dissemination The study will provide answers to important questions around the nature and process of reforms in institutional care that promote recovery while being cognizant of protecting human rights, and dignity. Ethical approval for SITAR was obtained from a registered ethics committee in India (Institutional Ethics Committee VikasAnvesh Foundation, VAF/201819/012 dated 6 December 2018) and the University of Warwick's Biomedical and Scientific Research Ethics Committee (REG0-2019-2332, dated 21 March 2019), and registered on the Central Trial Registry of India

\section{Strengths and limitations of this study}

- This is the first ever methodologically robust study in low-income and middle-income countries to test the impact of reforms in a psychiatric hospital on important patient outcomes such as change in disability, symptoms, social and occupational functioning and quality of life.

- The study offers an individual recovery plan for a psychiatric hospital setting in low resource settings.

- The cost implication of the individual service package will be studied; this has relevance in influencing mental healthcare policy across the country.

- There is a strong component of government involvement that adds to the potential of sustainability and scaling up across other psychiatric hospitals in the country.

- It is not possible to blind the case managers to the group allocation due to the nature of the intervention, hence it is a single-blind study, with only researchers assessing outcomes being blind to allocation.

- Given the nature of the setting, there is also a risk of contamination across both trial arms.

(CTRI/2019/01/017267). Trial results will be published inaccordance to CONSORT guidelines.

\section{INTRODUCTION}

People living with severe mental disorders (SMD) (psychosis, bipolar and affective disorders and severe-to-moderate depression) in low-income and middle-income countries (LMICs) face impediments to their clinical and functional recovery, and have large unmet needs associated with poverty, protection of human rights, social inclusion and participatory citizenship. ${ }^{1-5}$ A range of costeffective and evidence-based interventions 
are now available, however there are major barriers in access to appropriate care, increasing vulnerability and disadvantage along with stigma and discrimination. ${ }^{2}$ 6-9 Many languish in large hospitals, abandoned by family and forgotten by policy makers. India has 43 psychiatric hospitals built during the colonial period that continue to function almost in the same way as they did when they were set up. ${ }^{10-14}$ These hospitals constitute $80 \%$ of all available psychiatric beds. ${ }^{15}$ At the end of 2015, there were 6829 patients staying in 30 of the 43 psychiatric hospitals; $16 \%$ had been inpatients for $>5$ years, some for three to four decades. ${ }^{5}$ The infrastructure and standards of care are poor. ${ }^{14}$ There are no clear pathways to discharge and successfully integrate former patients into the community. ${ }^{16}$ A complex mix of low priority for mental healthcare in India, lack of support from central and state governments and low autonomy and decisionmaking power among professionals working in such institutions has impeded any meaningful reform. ${ }^{14}$

Psychiatric hospitals in India have played an important role in the care of very vulnerable people and continues to remain a legitimate and relevant locus of care for people in need of services. ${ }^{17}$ Given the lack of feasibility of closing down psychiatric institutions in most LMICs, there is an urgent need for manageable and evidencebased reform of these hospitals. The Udaan programme seeks to address this need.

\section{The Udaan programme}

Udaan is a partnership of Tata Trusts with government of Maharashtra, formalised through a Memorandum of Understanding, to develop the Regional Mental Hospital Nagpur (RMHN) as a centre of excellence through systematic reform of the hospital. Maharashtra is a state in the Western peninsular region of India with Nagpur being right in the centre of the country. Udaan (which in Hindi mean 'to soar') comprises four key reform elements: structural (refurbishing old colonial infrastructure to meet current service user needs), process (standardising clinical and non-clinical processes of the hospital), capacity building (standard training for different levels of hospital staff) and introduction of the needs-based intensive case management (NB-ICM), an individual need-based, recovery-oriented, service package for patients delivered through ICM. The Udaan elements are detailed in figure 1 .

\section{Structured Individualised inTervention And Recovery}

The Structured Individualised inTervention And Recovery (SITAR) study is embedded within the Udaan programme. In a clinical trial we test whether NB-ICM improves patient outcomes among long stay inpatients, in comparison to care as usual in a psychiatric hospital undergoing reform in an LMIC. The objectives of SITAR are:

a. To compare the effectiveness of structural and process reform with and without an individually tailored recovery plan on patient-level outcomes of disability (primary outcome), symptom severity, social and occupational functioning and quality of life for the long stay patient cohort of the hospital.

b. To determine the costing of implementing an individually tailored recovery plan for long stay individuals in psychiatric hospitals.

ICM calls for high resources and as such may not be feasible in low-income settings. We thus seek to compare patient outcomes emerging from larger structural and process reform in old psychiatric hospitals as compared with patient outcomes when ICM is added along with the reform. This comparison has significant value in policy decision making on how meagre resources should be used in low resource settings where mental healthcare continues to be provided by psychiatric hospitals set up 100 to 200 years ago.

This paper presents the protocol of SITAR, which aims to bridge a critical gap in scientific evidence by studying the impact of reform of psychiatric hospitals on individual patient outcomes. The findings will provide an evidencebased package of reforms for psychiatric hospitals in transition in LMICs.

\section{METHODS AND ANALYSIS}

\section{Study design and management}

The study is a pragmatic parallel-arm single-blind randomised controlled trial at a single site, the RMHN. The psychiatric hospital in Nagpur was started in 1864. The hospital has a capacity of 940 beds with an average occupancy of 600 patients at any given time.

Recruitment of patients for the study was initiated after completion of permissions, ethics approval and trial registry. We will continue recruitment till adequate sample size ( 85 in each arm) is reached. This is a changing population with a constant process of admission and discharge to the hospital. We assume a 6 months time frame to complete full recruitment from the start of intervention.

The inpatient population of the hospital will be compiled on a database, mapping sociodemographic variables, history of illness and history of treatment as baseline data. Patients fitting the inclusion criteria will be identified and randomly assigned to the intervention and control arms of the study. Recruitment will be continued till desired numbers are reached. The intervention will be carried out for a 6-month period. Postmeasures on all patients who have undergone premeasures will be undertaken at completion of intervention (at 6 months) and at two follow-up intervals postintervention of 9 and 12 months (3 and 6 months after completion of intervention). The SITAR study design is presented graphically in figure 2.

Several steps are proposed to ensure quality control and minimise the risk of bias:

1. Use of a standard case management intervention (intervention manual developed for the study).

2. Randomisation of the sample to intervention and care as usual arms of the study. 
Program design Udaan

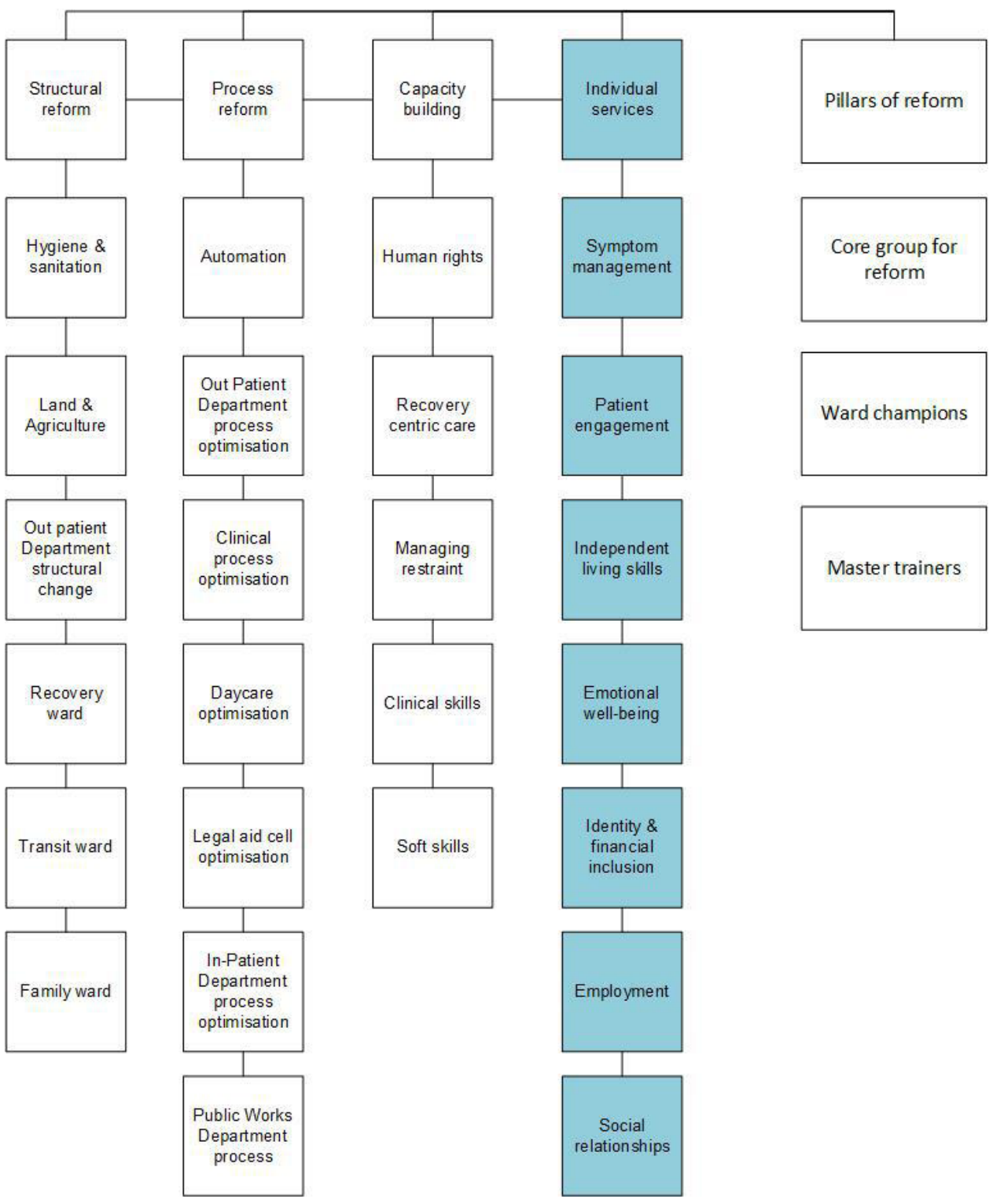

Indicates the intervention implemented as part of SITAR

Figure 1 Graphic representation of Structured Individualised inTervention And Recovery (SITAR).

3. Outcome measurements will be undertaken by researchers independent of the case managers delivering the intervention. Inter-rater reliability for the researchers will be computed. The statistician drawing the randomisation tables will be blinded to the allocation of the groups.

4. Each case manager will be supervised at least once every month on at least $20 \%$ of the cases undertaken by them. Joint monthly meetings of all case managers will be held for case reviews and sharing of experiences and discussion on overcoming barriers.

5. The primary supervisor will conduct a site visit and meet the case managers to assess fidelity of intervention.
Given the nature of the setting, there is a risk of contamination across arms especially since the hospital staff providing care in both arms are the same. We believe this is a minimal possibility given the meagerness of engagement of hospital staff with the patients.

SITAR is part of the work done by the first author in fulfilment of the $\mathrm{PhD}$ programme at the University of Warwick. The study will be coordinated by the Udaan office located at RMHN. The study is managed by the PI with supervision from the supervisors and oversight by the Trial Management Committee (TMC). The TMC comprises members from the University of Warwick and mental health experts from India. 
SITAR- A graphic representation of the research design

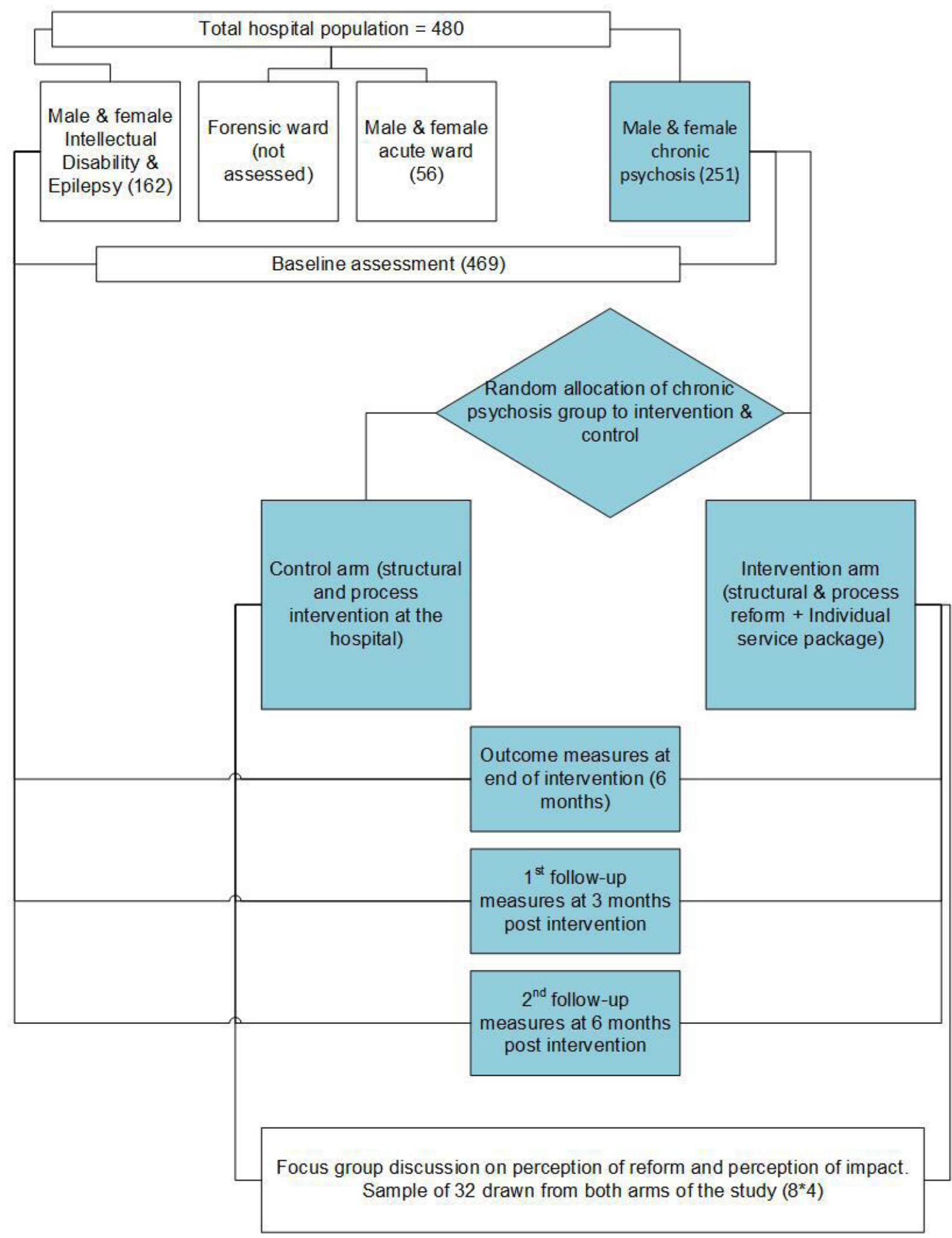

Indicates the 'two arm pragmatic randomised controlled trial'

Figure 2 Graphic representation of Udaan and Structured Individualised intTervention And Recovery (SITAR) 


\section{Participant eligibility and recruitment}

Inpatients at baseline will comprise all service users admitted to RMHN. Patients meeting the inclusion criteria for the study will be randomised to the two arms of the study. Service users will be eligible if they have a primary diagnosis of psychosis (schizophrenia, bipolar affective disorders and psychosis not otherwise specified) based on diagnosis given in their case files, a continued length of hospital stay between 12 and 120 months and are over the age of 18 years. Service users will be excluded from the study if they are over the age of 60 years, have a neurodevelopmental disorder such as epilepsy, an intellectual disability or are service users in acute and forensic wards.

\section{Sample size}

For the study to be powered at the $90 \%$ level with $5 \%$ significance level, the required sample is 170 people, 85 in each arm. Assuming a $15 \%$ dropout, we aim to recruit 100 people in each arm of the study. For the power calculation, the estimated sample size allows us to detect a minimum clinical difference of 10 points in the primary outcome (WHO Disability Assessment Scale (WHODAS)) at 6 months with an SD of 20. This equates to a moderate effect size of $0.5{ }^{18}$ The parameter estimates to inform the sample size were drawn from an Indian study based in the community with non-ICM using the WHODAS score as the primary outcome measure. ${ }^{19}$ People with psychosis in institutional set-ups might have higher disability levels as compared with people living in the community, however most people in LMICs continue to remain in institutions due to the absence of viable pathways of community reintegration. The intervention being offered is intensive with longer case management time than what would be feasible in a dispersed community setting.

\section{Informed consent}

The treating psychiatrist will assess the service users' ability to participate in the study as well as ability to consent. The psychiatrist will provide consent for those patients unable to give consent but deemed appropriate for the intervention. This is especially important in the case of this intervention since it is a 'need-based' psychosocial intervention. Based on inability to consent, patients who may need the intervention most might actually be left out of the study. The consent by the treating psychiatrist will ensure equitable inclusion. Additionally, the ward in charge also signs off on the consent. The study will be explained pictorially to the service user with the aid of a specially designed flip chart. Signatures and/or thumb impressions will be taken on simple consent forms drawn up in Hindi and Marathi. Service users will be assured that their refusal to participate/consent to the study will have no impact on the care they receive.

\section{Randomisation}

The study will use a computer-generated permuted block randomisation schedule for the allocation of recruited subjects to the two study arms. The researcher will create a list of service users meeting the inclusion criteria and consenting to the study and give them a unique ID number. This list will be handed over to the statistician who is independent to the research team. Random allocation of eligible study subjects to two study arms (A and B) will be done by the statistician using ralloc software (V.3.7.6) available in STATA (V.10.1, 2011) module.

\section{Intervention}

The NB-ICM for people living with SMD is based on a psychosocial rehabilitation model that takes a holistic approach to improving quality of life, reducing disability, improving role function, promoting independence and autonomy based on a hope for the future. It is a mix of working on individual competencies in the context of real everyday experiences and introducing environmental change propelled by individual choice. ${ }^{20-27}$

Trained case managers will deliver the intervention through a clinical and ICM approach that taps into a functional network of a spectrum of services being created at the hospital level through the reform process.

The case managers are trained on a specially designed training module that comprises content around severe mental illness and ICM with a focus on the needs of people with high disabilities in psychiatric institutions. The 60 hours training module was delivered through 7 days of offsite training for the purpose of this study.

The intervention components comprise (1) accommodation, safety and food (this is contextualised to the hospital setting where all service users may not have access to clean living spaces and enough food); (2) psychoeducation (about the illness and its symptoms); (3) symptom management; (4) physical health; (5) emotional wellbeing; (6) self-care and other living skills; (7) social relationships; (8) connecting with family; (9) leisure activities; (10) occupational and financial inclusion and (11) spiritual needs.

The intervention is based on an objective assessment of current needs of the service user and provides a comprehensive package of services to meet the range of individual needs. The case manager works collaboratively with the person in developing a personalised care plan drawing from the larger context of available opportunities within the hospital, created through the ongoing reform process. The care plan adjusts to the patient's cultural context. It draws on the strengths and potential of the individual and is focused on the reduction of personal distress and disability. Care provided through this approach is continuous and consistent for the defined period of the intervention. Case managers have the primary responsibility for planning, coordinating and delivering the care. Each case manager will have a caseload of 12-14 service users and spend at least eight sessions per case per month. The case manager will deliver the intervention face-to-face either in the ward complex or through calls and home visits in case the person is discharged from hospital as per protocol. 
The intervention will aim:

- To address unmet needs on symptomatology through appropriate pharmacological management and psychosocial support. It also includes diminishing and eliminating wherever possible the adverse physical and behavioural consequence of symptom management as well as those arising out of prolonged institutionalisation.

- To address unmet basic needs of adequate accommodation and food.

- To address unmet needs on personal functioning, improving activities of daily living both in terms of skills and access to opportunities.

- To address unmet needs of social connectedness, engagement, leisure and social competence through individual competency building and access to environmental opportunities.

- To address unmet needs for personal identity and citizenship.

- To address the unmet needs of occupational functioning, employment and financial inclusion.

- To address the unmet needs of connecting to family and community where feasible.

Patients in the control arm will go through the same baseline and follow-up measurements as the intervention arm. This group will however not receive the NB-ICM during the trial period, the control arm will continue receiving care as usual, in this case care being provided in a setting undergoing reform. In most psychiatric hospitals in India, care as usual largely comprises biomedical management. ${ }^{1428}$

The intervention will be discontinued given the following conditions: (1) if the participant wants to discontinue participation; (2) an acute illness episode that significantly disrupts time in intervention (beyond 4 weeks); (3) when the participant is discharged from the hospital and community-based intervention is not possible either due to distance beyond Nagpur district, unwillingness of participant or family for home-based intervention; (4) in case of death of a participant.

\section{Adverse events: recording and reporting}

Given the nature of the study population and the chronicity of the illness certain events are expected. The study protocol classifies these events under 'adverse events' and 'serious adverse events'. Adverse events comprise (a) acute illness (psychosis) episodes as determined by transfer to acute ward; (b) episodes of isolation and restraint; (c) transfer for medical care outside the psychiatric hospital; (d) absconding from the facility. Serious adverse events comprise (e) episode of self-harm and (f) death.

To record and report adverse events, we will use the Warwick CTU's Clinical Trials Standard Operating Procedure 17 part 2 Safety Reporting for Clinical Trials other than those of Investigational Medicinal Products V.1.5.

Any adverse event occurring with any participant will be first notified and discussed with the ward in charge. Based on routine hospital care processes, it is the responsibility of the ward in charge to initiate action of either directly providing any care, making a psychiatric referral or making a medical referral. All recorded adverse events will be reported to the core committee and the trial supervisor through monthly reports. These reports will also be submitted to the TMC. Any unexpected adverse event will be reported to Tata Trusts (as the sponsor) along with the India ethics committee, the Central Trial Registry of India as well as the university ethics committee (BSREC) within 15 days of the event.

\section{Measurements}

The study comprises outcome measures and process measures. Other baseline measurements include sociodemographic details, illness history and treatment history.

\section{Outcome measures}

Assessment of level of disability will form the primary outcome for the study. WHODAS V.2.0, a generic assessment instrument for health and disability that produces standardised disability levels and profiles applicable across cultures and diseases. ${ }^{29}$ SITAR will use the simple scoring format sufficient to describe the degree of functional limitation. ${ }^{29}$ Three items of WHODAS are not applicable for scoring due to the nature of the setting. These are items 3.4, 4.5 and 6.6. Secondary outcome measures include an assessment of severity of symptoms, assessment of social and occupational functioning and assessment of quality of life. The scales used for these measurements include the symptom measure-The Clinical Global Improvement Scale (Schizophrenia) (CGI-S), a brief, stand-alone assessment of the clinician's view of the patient's global functioning prior to and after initiating a study medication or intervention. ${ }^{30}$ The CGI comprises two one-item measures evaluating (a) severity of psychopathology from 1 to 7 and (b) change from the initiation of treatment on a similar 7-point scale. ${ }^{31}$ Social and Occupational Functioning Scale assesses individual's level of social and occupational functioning and is not directly influenced by the overall severity of the individual's psychological symptoms. ${ }^{32} 33$ Health-related quality of life measure EuroQol-5D (EQ-5D) is a widely used generic patient-reported outcome questionnaire designed specifically for cost-utility economic evaluation internationally. The EQ-5D asks patients to indicate whether they have no, some or extreme problems on each of five dimensions of health: mobility; self-care; usual activities; pain/discomfort and anxiety/depression. ${ }^{34} 35$

Process (intervention) measurements include the assessment of need through a standard form based on Camberwell Assessment of Need. ${ }^{36}$ The adaptation draws from prior use of this measure in India through the formative study of needs ${ }^{37}$ and need assessment formats used in community setting. ${ }^{38}$ This will be carried out by the allocated case managers five times during the study period and will be an indicator of the number of met and unmet needs of the service user at different points during the study. The intervention plan, case managers will draw up a personal care plan collaboratively with the service user 
and the ward in charge on a monthly basis. Case managers will record the plan on a standard form developed for the intervention and reviewed monthly by the researcher. The symptoms checklist has been adapted from ones used in other Indian settings and will record the change in symptoms over the study period and serve as an adjunct to the symptom measure (CGI). The case manager will carry out the measure five times during the study period. Self-care and other living skills checklist is adapted to an institutional setting from scales for assessing activities of daily living. ${ }^{39}{ }^{40}$ Its purpose is to aid the case manager in assessing progress on the intervention plan.

\section{Baseline and follow-up measurements schedule}

Baseline measurements will be initiated at the start of the study and completed for all inpatients over a 3-month period by trained research assistants (RA) who have a Master's degree in Psychology or Social Work RAs are not involved in the hospital setting, however unmasking is possible and we will record all episodes of unmasking. Inter-rater reliability will be established for all the RAs conducting the measurements.

The intervention will be initiated after completion of the baseline measurements and carried out for a period of 6 months. At the end of the 6-month intervention period, the first outcome measurement will be initiated and completed over a 2-month period. The first and second follow-up outcome measurements will be initiated at 3 and 6 months postintervention, respectively, and completed over a 2-month period.

The patient sequence will be kept standard for the measurements to ensure uniformity in time between measures. In case of an adverse event where the patient may not be available for measurement as per sequence, accommodation will be made to complete the measure any time during the 2-month period of that measurement cycle. In case this is not possible, the patient will be considered as lost to follow-up. Sequence and time frame of measures are summarised in table 1. All patients (except dropouts as per criteria) will be followed up as per protocol either within the hospital or in the community.

\section{Qualitative element of the study}

The qualitative component of the study comprises patient perceptions on the overall reform process and the individual intervention and its felt impact. The SITAR study will use focus group discussions (FGDs) to elicit this. Four FGDs of 8 patients each (16 patients from each study arm) will be conducted in the last quarter of the study period. Since we seek to understand the lived experience of the service user, a phenomenological epistemological perspective is proposed. A basic thematic analysis will be done using NVivo. ${ }^{41}$ Verbatim quotes will also be used to highlight findings from quantitative components of the study.

\section{Data management and analysis}

Data collection

Quantitative data will be collected by trained RAs using predesigned, pretested tools as included in the protocol. Senior RA will check completeness and accuracy of data gathered on daily basis before electronic data entry.

\section{Data storage}

The paper data will be stored in secure cabinets, in the PI's cabin at the Tata Trust office in the hospital campus. The office is under CCTV surveillance. The data will be held for 10 years postcompletion of the study.

Table 1 List and time frame for assessments ( 6 months considered from date of first intervention)

\begin{tabular}{|c|c|c|c|c|c|c|c|c|c|c|c|c|c|c|c|}
\hline & & & \multicolumn{13}{|c|}{ Months } \\
\hline Assessment & Type & By & 0 & 1 & 2 & 3 & 4 & 5 & 6 & 7 & 8 & 9 & 10 & 11 & 12 \\
\hline WHODAS (disability) & $\mathrm{OM}$ & RA & ? & & & & & & 0 & & & ○ & & & 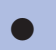 \\
\hline SOFS & OM & RA & - & & & & & & • & & & ○ & & & - \\
\hline CGI (symptoms) & OM & RA & - & & & & & & - & & & - & & & - \\
\hline EQ-5D (quality of life) & OM & RA & - & & & & & & - & & & - & & & - \\
\hline Episodes of seclusion and restraint & $\mathrm{OM}$ & $\mathrm{CM}$ & 0 & $\bullet$ & $\bullet$ & $\bullet$ & $\bullet$ & 0 & 0 & - & 0 & ○ & 0 & 0 & 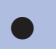 \\
\hline Discharge/adverse events & $\mathrm{OM}$ & $\mathrm{CM}$ & $\bullet$ & $\bullet$ & $\bullet$ & $\bullet$ & $\bullet$ & $\bullet$ & $\bullet$ & $\bullet$ & $\bullet$ & $\bullet$ & $\bullet$ & $\bullet$ & $\bullet$ \\
\hline Needs assessment & PM & $\mathrm{CM}$ & ? & & & 0 & & & 0 & & & ○ & & & 0 \\
\hline Intervention plan & PM & $\mathrm{CM}$ & $\bullet$ & $\bullet$ & $\bullet$ & ○ & $\bullet$ & $\bullet$ & $\bullet$ & & & & & & \\
\hline Symptoms checklist & PM & $\mathrm{CM}$ & $\bullet$ & & & - & & & $\bullet$ & & & $\bullet$ & & & $\bullet$ \\
\hline Self-care and other living skills checklist & PM & $\mathrm{CM}$ & $\bullet$ & $\bullet$ & • & 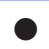 & ○ & $\bullet$ & - & & & & & & \\
\hline Case management record form & PM & $\mathrm{CM}$ & 0 & 0 & 0 & - & 0 & - & - & & & & & & \\
\hline
\end{tabular}

-, intervention time frame; CGI, Clinical Global Improvement Scale; CM, case manager; EQ-5D, EuroQol-5D; OM, outcome measure; PM, process measure; RA, research assistant; SOFS, Social and Occupational Functioning Scale; WHODAS, WHO Disability Assessment Scale. 


\section{Data entry and coding}

Data will coded and entered in an efficient database using MS Excel. Data will be kept confidential and anonymous on password-protected files. The master sheet will be kept separately on MS Excel with password protection. Built in validity, checks will be incorporated in data entry software with flash/warning alerts for incorrect or out of range values.

\section{Data screening, data validation and data editing}

Data will be screened at every stage, that is, prerandomisation, postrandomisation and closing stage of the trial. This will be done for each and every item of the individual record by student researcher (trial PI). Accuracy of electronic data will be checked through comparison with questionnaire data on a sample basis.

\section{Data analysis}

All results from the trial will be reported according to the Consolidation Standards of Reporting Trials (CONSORT) guideline for randomised controlled trials. ${ }^{42}$ Descriptive statistics will be presented for participant characteristics and outcomes collected in the trial summarised by treatment arm. Continuous outcomes will be summarised as mean and SD, categorical data will be summarised using frequency and percentage. If data are non-normal, the median and IQR will be presented.

All of the analyses will be based on the intention-to-treat principle, where a $\mathrm{p}$ value $<0.05$ will be considered as statistically significant. The primary analysis will estimate the treatment effect and $95 \%$ CI for the primary outcome (WHODAS) at the 6-month time point using a linear regression model having adjusted for clinically important baseline variables. For all secondary analyses, treatment effects will be estimated using adjusted linear regression models for continuous outcomes and adjusted logistic regression models for binary outcomes. The analyses will be undertaken at each of the follow-up time points (ie, 6,9 and 12 months). Where the assumption of normality is not valid, equivalent non-parametric alternatives (eg, rank-based statistics) will be used.

Every effort will be made to ensure that missing data are kept to a bare minimum in the trial. The level or extent of missingness in data will be assessed at the end of the trial, and if required, additional sensitivity analyses will be undertaken using multiple imputation techniques to impute the missing values.

\section{Data processing}

Data will be processed by the Udaan programme at baseline (prerandomisation), during trial (postrandomisation) and closing stage (outcome assessment). Raw data from the master file will be coded and processed into a data file. The entire data set will be put through an Excel-based double entry process by two independent people. Formula-based comparison of the two sets will be undertaken and discrepancies will be resolved by rechecking with the hard copy of the questionnaire on file. The data file will include both original variables as well as some newly derived variables or transformed variables specific to the study objectives. Statistical Package of Social Sciences will be used for data analysis. The data analyst will be blinded to treatment assignment.

\section{Anonymising data}

Direct identifiers that allow the identification and communication with an individual participant will be removed. The names of all participants will be replaced with a master list identity (ID) number. The master list containing the ID number will be kept with the PI on a password-protected file, which will be housed in a password-protected firewalled system. The data set for analysis will not include any email address, telephone numbers or home address of patients (where available). Quasi identifiers such as ward numbers will be removed from and variables such as date of admission and date of discharge will be generalised into length of stay.

\section{PATIENT AND PUBLIC INVOLVEMENT}

FGDs are built into the design of the programme to incorporate the patient experience of intervention. No patients were involved in the study design.

\section{COSTING AND POTENTIAL ECONOMIC GAINS OF THE INTERVENTION}

The SITAR study will also include a retrospective bottom-up cost analysis of the individualised intervention in terms of resource or input requirement along with costing of resources for care as usual. Cost elements will include all the resources used in development of the intervention and training material, costs of training, costs of intervention delivery which will include staff time and costs of supervision (people, facilities, equipment and supplies). The costing will be based on actual expenditure incurred through the Udaan programme as well as costs components derived through collaboration which include costs of items received in kind such as clothes, soaps and shampoos directly linked to patient care. Actual government spending on patient care will also be done. Costing will be appropriately apportioned to the SITAR study in terms of time allocation of staff based on an analysis of case management records. Given the scope of the study, costs will primarily be presented alongside measures of benefit listed in table 1 in the form of a cost-consequence analysis. ${ }^{43}$ A secondary costeffectiveness analysis will additionally be performed in which the trial primary outcome will be the measure of effectiveness. $^{44}$

\section{Ethical approval and dissemination}

Ethical approval for SITAR has been obtained from a registered ethics committee in India (Institutional Ethics Committee VikasAnvesh Foundation, VAF/201819/012 dated 6 December 2018) and the University of Warwick's Biomedical and Scientific Research Ethics 
Committee (REGO-2019-2332, dated 21 March 2019), and registered on the Central Trial Registry of India (CTRI/2019/01/017267).

Findings of the study will be presented through scientific publications as well as through a national-level dissemination in India along with presentations in different conferences. We also intend to do a policy paper recommending a feasible reform process for psychiatric hospitals in India. Trial results will be published in accordance with CONSORT guidelines.

\section{CURRENT TRIAL STATUS}

Recruitment of patients was initiated in April 2019. Recruitment was closed in December 2019. The final patient recruited will reach end point follow-up in December 2020.

\section{Author affiliations}

${ }^{1}$ Mental Health, Tata Trusts, Mumbai, India

${ }^{2}$ Mental health and wellbeing, Warwick Medical School, University of Warwick, Coventry, UK

${ }^{3}$ Center for Health Economics, Warwick Medical School, University of Warwick, Coventry, UK

${ }^{4}$ Warwick Cinical Trials Unit, University of Warwick, Coventry, UK

${ }^{5}$ Department of Psychiatry, NIMHANS, Bangalore, Karnataka, India

${ }^{6}$ Director, Centre for Mental Health and Wellbeing Research, Warwick Medical School, Coventry, UK

Contributors TR: Principal Investigator, design and implementation of the trial. Wrote first draft of protocol paper. HT: supported the design, protocol development, ethics application and continued supervision, review and editing of manuscript. JM: supported the design of economic elements of the protocol. DM: supported the development of the data recording and analysis plan. SJ: reviewed and edited the manuscript. SS: primary supervisor on the trial, supported the design, protocol development, ethics application for India and UK, Trial registry, ongoing supervision on the trial. All coauthors accepted the final version of the paper.

Funding This work is supported by Tata Education and Development Trust (TEDT) (DI Regional mental hospital Nagpur, circular number 130) and R G Manudhane Foundation for Excellence (MoU with TEDT dated 10 August 2016).

Competing interests This trial is part of the $\mathrm{PhD}$ programme undertaken by the TR. She is an employee of the Tata Trusts and the Tata Trusts External Individual educational grants programme funds the $\mathrm{PhD}$ won on basis of merit.

Patient and public involvement Patients and/or the public were not involved in the design, conduct, reporting or dissemination plans of this research.

Patient consent for publication Not required.

Provenance and peer review Not commissioned; externally peer reviewed.

Open access This is an open access article distributed in accordance with the Creative Commons Attribution Non Commercial (CC BY-NC 4.0) license, which permits others to distribute, remix, adapt, build upon this work non-commercially, and license their derivative works on different terms, provided the original work is properly cited, appropriate credit is given, any changes made indicated, and the use is non-commercial. See: http://creativecommons.org/licenses/by-nc/4.0/.

\section{ORCID iDs}

Tasneem Raja http://orcid.org/0000-0002-5821-8673

Helena Tuomainen http://orcid.org/0000-0003-1636-8187

Swaran Singh http://orcid.org/0000-0003-3454-2089

\section{REFERENCES}

1 Chatterjee S, Patel V, Chatterjee A, ea CS, et al. Evaluation of a community-based rehabilitation model for chronic schizophrenia in rural India. Br J Psychiatry 2003;182:57-62.
2 Patel V, Chisholm D, Parikh R, et al. Addressing the burden of mental, neurological, and substance use disorders: key messages from disease control priorities, 3rd edition. Lancet 2016;387:1672-85.

3 Ernest S, Nagarajan G, Jacob KS. Assessment of need of patients with schizophrenia: a study in Vellore, India. Int J Soc Psychiatry 2013;59:752-6.

4 Kulhara P, Avasthi A, Grover S, et al. Needs of Indian schizophrenia patients: an exploratory study from India. Soc Psychiatry Psychiatr Epidemiol 2010;45:809-18.

5 Gururaj G, Varghese M, Benegal V, et al. National mental health survey of India, 2015-2016: prevalence, patterns and outcomes. Bengaluru: National Institute of Mental Health and Neuro Sciences, 2016.

6 Kohn R, Saxena S, Levav I, Saraceno B, et al. The treatment gap in mental health care. Bull World Health Organ 2004;82:858-66.

7 Saxena S, Thornicroft G, Knapp M, et al. Resources for mental health: scarcity, inequity, and inefficiency. Lancet 2007;370:878-89.

8 Patel V. Mental health in low- and middle-income countries. $\mathrm{Br} \mathrm{Med}$ Bull 2007;81-82:81-96.

9 Vos T, Barber RM, Bell B, et al. Global, regional, and national incidence, prevalence, and years lived with disability for 301 acute and chronic diseases and injuries in 188 countries, 1990-2013: a systematic analysis for the global burden of disease study 2013. The Lancet 2015;386:743-800.

10 Ernst W. Crossing the boundaries of 'colonial psychiatry'. Reflections on the development of psychiatry in British India, C. 1870-1940. Cult Med Psychiatry 2011;35:536-45.

11 Lin C-Y, Huang A-L, Minas H, et al. Mental hospital reform in Asia: the case of Yuli veterans Hospital, Taiwan. Int J Ment Health Syst 2009;3:1.

12 Channabasavanna S, Murthy P. The National human rights Commission report 1999: a defining moment. New Delhi: Elsevier, 2004.

13 Jain S. Psychiatry and confinement in India. The Confinement of the Insane: International Perspectives, 2003: 1800-965.

14 Murthy P, Kumar S, Desai N, et al. Mental health care in India-old aspirations, renewed hope. National Human Rights Commission: Report of the Technical Committee on Mental Health New Delhi, 2016.

15 World Health Organization. World mental health atlas, 2014.

16 NHRC. Care and treatment in mental health institutions; some glimpses in the recent period. India: National Human Rights Comission, 2012.

17 Murthy P, Isaac M, Dabholkar H. Mental hospitals in India in the 21st century: transformation and relevance. Epidemiol Psychiatr Sci 2017;26:10-15

18 Cohen J. Statistical power analysis for the behavioral sciences, 1988

19 Srinivasa Murthy R, Kishore Kumar KV, Chisholm D, Murthy RS, Kumar KK, et al. Community outreach for untreated schizophrenia in rural India: a follow-up study of symptoms, disability, family burden and costs. Psychol Med 2005;35:341-51.

20 Wolfson P, Holloway F, Killaspy $\mathrm{H}$. Enabling recovery for people with complex mental health needs: a template for rehabilitation services. Royal College of Psychiatrists, 2009.

21 World Health Organization. Psychosocial rehabilitation: a consensus statement. Int J Ment Health 1997;26:77-85.

22 Anthony WA, Cohen MR, Farkas MD, et al. Psychiatric rehabilitation. MA: Center for Psychiatric Rehabilitation, Boston University, Sargent College of Allied Health Professions Boston, 1990.

23 Dieterich M, Irving CB, Park B, et al. Intensive case management for severe mental illness. Cochrane Database Syst Rev 2010:CD007906.

24 Kopelowicz A, Wallace CJ, Liberman RP. Psychiatric rehabilitation. In: Gabbard GO, ed. Treatments of psychiatric disorders. 4th ed. Arlington, VA: American Psychiatric Publishing Inc, 2007: 361-78.

25 Liberman RP, Kopelowicz A. Recovery from schizophrenia: a challenge for the 21st century. Int Rev Psychiatry 2002;14:245-55.

26 Marshall M, Gray A, Lockwood A, et al. Case management for people with severe mental disorders. Cochrane Database Syst Rev 2000:CD000050.

27 Solomon P. The efficacy of case management services for severely mentally disabled clients. Community Ment Health J 1992;28:163-80.

28 Varma S. Disappearing the asylum: Modernizing psychiatry and generating manpower in India. Transcult Psychiatry 2016;53:783-803.

29 Üstün TB. Measuring health and disability: manual for who disability assessment schedule WHODAS 2. World Health Organization, 2010.

30 Haro JM, Kamath SA, Ochoa S, et al. The clinical global ImpressionSchizophrenia scale: a simple instrument to measure the diversity of symptoms present in schizophrenia. Acta Psychiatr Scand 2003;107:16-23.

31 Busner J, Targum SD. The clinical global impressions scale: applying a research tool in clinical practice. Psychiatry 2007;4:28-37. 
32 Morosini PL, Magliano L, Brambilla L, et al. Development, reliability and acceptability of a new version of the DSM-IV social and occupational functioning assessment scale (SOFAS) to assess routine social functioning. Acta Psychiatr Scand 2000;101:323-9.

33 Saraswat N, Rao K, Subbakrishna DK, et al. The social occupational functioning scale (SOFS): a brief measure of functional status in persons with schizophrenia. Schizophr Res 2006;81:301-9.

34 Devlin N, Shah K, Feng Y, et al. Valuing health-related quality of life: an EQ-5D-5L value set for England, 2016.

35 Janssen MF, Pickard AS, Golicki D, et al. Measurement properties of the EQ-5D-5L compared to the EQ-5D-3L across eight patient groups: a multi-country study. Qual Life Res 2013;22:1717-27.

36 Slade M. CAN: Camberwell assessment of need: a comprehensive needs assessment tool for people with severe mental illness. Gaskell, 1999.

37 Balaji M, Chatterjee S, Brennan B, et al. Outcomes that matter: a qualitative study with persons with schizophrenia and their primary caregivers in India. Asian J Psychiatr 2012;5:258-65.
38 Chatterjee S, Naik S, John S, et al. Effectiveness of a community-based intervention for people with schizophrenia and their caregivers in India (COPSI): a randomised controlled trial. Lancet 2014;383:1385-94.

39 Shelkey M, Wallace M. Katz index of independence in activities of daily living. Home Healthc Nurse 2001;19:323-4.

$40 \mathrm{Graf} \mathrm{C}$. The Lawton instrumental activities of daily living scale. Am J Nurs 2008;108:52-62.

41 Denardo AM. Using NVivo to analyze qualitative data, 2002.

42 Schulz KF, Altman DG, Moher D, et al. CONSORT 2010 statement: updated guidelines for reporting parallel group randomised trials. BMJ 2010;340:c332.

43 Mauskopf JA, Paul JE, Grant DM, et al. The role of costconsequence analysis in healthcare decision-making. Pharmacoeconomics 1998;13:277-88.

44 Husereau D, Drummond M, Petrou S, et al. Consolidated health economic evaluation reporting standards (cheers) statement. Int $J$ Technol Assess Health Care 2013;29:117-22. 